\title{
Escala de Desenvolvimento de Carreira de Universitários: Estudo Confirmatório com Estudantes Portugueses
}

\author{
Joana Soares ${ }^{1}$, Maria do Céu Taveira ${ }^{1}$, Íris M. Oliveira ${ }^{2}$, Marina Cardoso de Oliveira ${ }^{3}$, Lucy \\ Leal Melo-Silva ${ }^{4} \&$ Marco Antônio Pereira Teixeira ${ }^{5}$ \\ ${ }^{1}$ Escola de Psicologia, Universidade do Minho \\ ${ }^{2}$ Escola Superior de Educação de Fafe, Instituto de Estudos Superiores de Fafe \\ ${ }^{3}$ Instituto de Educação, Letras, Artes, Ciências Humanas e Sociais, Universidade Federal do Triângulo Mineiro \\ ${ }^{4}$ Faculdade de Filosofia, Ciências e Letras de Ribeirão Preto, Universidade de São Paulo \\ ${ }^{5}$ Instituto de Psicologia, Universidade Federal do Rio Grande do Sul
}

\begin{abstract}
Resumo: A contemporaneidade, marcada por um mercado de emprego flexível e competitivo, exige que os jovens, nomeadamente universitários, sejam capazes de gerir a sua carreira. Para que os profissionais de carreira consigam auxiliar estes jovens, precisam de ter à sua disposição instrumentos válidos e fiáveis que lhes possibilitem avaliar comportamentos de carreira, como as respostas adaptativas. Este estudo tem por objetivo analisar as propriedades psicométricas da Escala de Desenvolvimento de Carreira de Universitários numa amostra de universitários portugueses. Participaram 216 estudantes divididos em duas subamostras: subamostra $A$ com 111 estudantes entre os $19-36$ anos $(\mathrm{M}=22.67$, $\mathrm{DP}=2.56)$, e subamostra B com 105 estudantes entre os $19-36$ anos $(\mathrm{M}=22.42$, DP $=2.08)$. As análises fatoriais exploratórias indicam solução penta-fatorial com boa consistência interna, após se eliminarem quatro itens. As análises fatoriais confirmatórias indicam ajustamento moderadamente aceitável para o modelo penta-fatorial. Mais estudos psicométricos com a medida são necessários.
\end{abstract}

Palavras-chave: Desenvolvimento de carreira; respostas adaptativas; universitários; medida.

University Career Development Scale: Confirmatory Study with Portuguese Students: Contemporaneity, characterized by a flexible and competitive job market, requires that young people, namely college students, are able to manage their careers. For career professionals to be able to help young people, valid and reliable instruments are needed to evaluate career behaviors, such as adaptative responses. This study sought to analyze the psychometric properties of the University Career Development Scale on a sample of Portuguese college students. Participants included 216 students divided into two subsamples: sub-sample A with 111 students aged 19-36 ( $\mathrm{M}=22.67, \mathrm{SD}=2.56)$, and sub-sample B with 105 students aged 19-36 $(\mathrm{M}=22.42, \mathrm{SD}=2.08)$. Exploratory factor analyses indicate penta-factorial solution, with good internal consistency, after eliminating four items. Confirmatory factor analyses indicate a moderately acceptable fit for the penta-factorial model. Further psychometric studies with the measure are necessary.

Keywords: Career development; confirmatory factor analysis; college students; adaptative responses; measurement.

A quarta revolução industrial emerge na contemporaneidade com um mercado de emprego competitivo e instável, exigindo aos indivíduos uma maior flexibilidade e capacidade de resolução de problemas (World Economic Forum, 2018). Este novo paradigma, substituto do paradigma de uma carreira estável e para a vida, exige que as pessoas sejam capazes de gerir a sua própria carreira ao longo do tempo (Wilhelm \& Hirschi, 2019). Uma fase particularmente importante e influente no processo de construção da carreira é a passagem para o mercado de emprego (Schoon \& Silbereisen, 2009). Neste enquadramento, a literatura sugere que muitos jovens, nomeadamente diplomados do ensino superior, podem não estar preparados para lidar com um mercado de emprego incerto e competitivo, o que se reflete na dificuldade em tomar decisões de carreira e, consequentemente, inserir-se profissionalmente (e.g., Oliveira, 2014; Oliveira \& Dela Coleta, 2008; Perrone \& Vickers, 2003; Sidiropoulou-Dinakakou et al., 2012; Teixeira \& Gomes, 2004). 0 processo de transição para uma nova etapa de carreira é muitas vezes encarado, por estes diplomados, com uma dualidade de sentimentos. Por um lado, sentem alegria e satisfação por conseguirem finalizar a

${ }^{1}$ Morada para correspondência: Maria do Céu Taveira, Escola de Psicologia, Universidade do Minho, Campus de Gualtar, 4710-057, Braga, Portugal. Email: ceuta@psi.uminho.pt 
graduação (Teixeira \& Gomes, 2004), por outro, sentem stress, medo, incerteza, perda e baixa confiança, aquando do momento de entrada no mercado de emprego (Perrone \& Vickers, 2003; Postigo \& Oliveira, 2015). Estes sentimentos podem justificar-se pela incapacidade de os estudantes moldarem o meio, ou seja, gerirem a sua vida no sentido de alcançar os objetivos de carreira pré-estabelecidos (Oliveira et al., 2017). Este processo parece despoletar o questionamento e a reflexão dos estudantes, sobre quem são, onde estão e para onde querem ir (Perrone \& Vickers, 2003). Como tal, o processo de transição para o mercado de emprego parece afetar a identidade destes recém-formados que, por um lado, deixam de ser estudantes, mas, por outro, ainda não são trabalhadores (Oliveira, 2014; Perrone \& Vickers, 2003; Teixeira \& Gomes, 2004). Assim, dois racionais úteis e complementares que permitem compreender o processo de transição universidade-mercado de emprego em universitários são a Teoria de Construção de Carreira (TCC, Savickas, 2005) e a Teoria Sociocognitiva da Carreira (TSCC, Lent \& Brown, 2013; Lent et al., 1999). Tratase de teorias explicativas que identificam fatores psicossociais responsivos e maleáveis aos desafios desenvolvimentistas e contextuais que a transição universidade-mercado de emprego impõe. Para além disso, explicam a maior ou menor presença - ou ativação -, de comportamentos de carreira adaptativos (e.g., planeamento, formulação de objetivos, recolha de informação, negociar transições) ao longo do ciclo de vida.

A TCC (Savickas, 2005; Savickas, 2002) surge como uma atualização da Teoria de Desenvolvimento de Carreira de Donald Super (1980-1990) face ao contexto laboral mais competitivo e instável do século XXI. Por um lado, a TCC substitui o conceito de maturidade vocacional (Super, 1980) pelo conceito de adaptabilidade de carreira (Savickas, 2005), um constructo psicossocial que representa a prontidão e os recursos que cada indivíduo possui para resolver tarefas vocacionais e/ou ocupacionais (Savickas, 2013). Por outro, passa a conceptualizar o conceito de carreira como um processo de construção contínuo, em que as pessoas se preparam com tempo, transitam para uma nova fase de vida e vão constantemente adaptando-se a meio, de forma a se integrarem socialmente (e.g., Ambiel, 2014; Bardagi \& Albanaes, 2015; Savickas \& Porfeli, 2012). Em 2012, Savickas aprofunda a sua teoria e propõe que a adaptação ao longo da carreira seja definida a partir de quatro componentes, variantes da adaptabilidade. São elas: a prontidão adaptativa, os recursos adaptativos, as respostas adaptativas e os resultados de adaptação. A prontidão adaptativa é definida como uma variável de tipo traço que caracteriza a flexibilidade para lidar com a mudança. Esta componente tem sido medida através de constructos como a autoestima, a autoavaliação e a escala de traços básicos de personalidade Big-Five (e.g., Hirshi et al., 2015; Rudolph et al., 2017). Os recursos adaptativos - constructo de natureza psicossocial -, define ferramentas cognitivo-motivacionais que facilitam a resolução de tarefas vocacionais, como é o caso da adaptabilidade. A adaptabilidade, segunda componente no modelo, de acordo com Savickas e Porfeli (2012), inclui as dimensões de preocupação, curiosidade, controlo e confiança em relação ao futuro. Este constructo tem sido medido sobretudo com a Escala sobre Adaptabilidade de Carreira (e.g., Duarte et al., 2012; Gomes, 2017; Hirshi et al., 2015; Monteiro \& Almeida, 2015; Rudolph et al., 2017). A terceira componente, respostas adaptativas, designa comportamentos e atitudes que permitem lidar com transições de carreira, sendo frequentemente medida por escalas de planeamento, exploração, autoeficácia e comportamentos de tomada de decisão (e.g., Hirshi et al., 2015; Rudolph et al., 2017), daqui em diante designados por comportamentos de carreira adaptativos. Finalmente, a quarta componente, os resultados adaptativos, refletem uma maior ou menor satisfação de carreira, sucesso, envolvimento no trabalho, empregabilidade percebida, entre outros (e.g., Gomes, 2017; Oliveira, 2014; Pinheiro, 2017; Rudolph et al., 2017; Savickas \& Porfeli, 2012). De acordo com a teoria e evidência empírica, a adaptação na carreira segue uma sequência na qual pessoas que apresentam maior volição para a mudança (prontidão adaptativa) apresentarão também maior eficiência no processo autorregulatório (recursos adaptativos) e, consequentemente, mais comportamentos e atitudes (respostas adaptativas) que lhes permitem adaptar-se ao meio (resultados adaptativos) (e.g., Hirshi et al., 2015; Rudolph et al., 2017; Savickas \& Porfeli, 2012; Savickas et al., 2018). De facto, a literatura é consensual em afirmar que apesar de independentes, as quatro componentes do modelo de adaptação na carreira (Savickas \& Porfeli, 2012) se relacionam entre si (e.g., Gomes, 2017; Hirshi et al., 2015; Pinheiro, 2017, Rudolph et al., 2017).

Por seu turno, a TSCC (Lent et al., 1999; Lent \& Brown, 2013), tal como a teoria anterior, surge como uma evolução teórica, mas neste caso ancorada na Teoria Sociocognitiva da Aprendizagem de Bandura (1986). Segundo a TSCC, a adoção de comportamentos de carreira adaptativos (e.g., procura de emprego, estabelecimento de objetivos de vida) dependem do sentido de autoeficácia, das expectativas de resultado e dos objetivos estabelecidos (Lent et al., 1999; Lent \& Brown, 2013). A autoeficácia, primeira componente, define-se como a crença acerca das capacidades individuais para alcançar um objetivo ou comportamentos específicos pretendidos (Lent et al., 1999). As expectativas de resultado, segunda componente, representam as crenças individuais sobre as consequências de determinado comportamento (Lent et al., 1999). Como tal, se uma pessoa acreditar que é capaz de realizar um determinado comportamento com 
sucesso e este lhe trouxer consequências positivas e desejadas, a probabilidade de despender tempo e esforço no mesmo aumenta. Os objetivos, terceira componente, representam a determinação individual para se envolver num dado comportamento (Lent et al., 1999). A par destas três componentes principais, os autores da TSCC destacam ainda a importância que o suporte social e as barreiras contextuais podem ter na adoção de comportamento de carreira adaptativos (Lent et al., 1999; Lent \& Brown, 2013). Como tal, a perceção de uma rede de suporte alargada (e.g., família, amigos), que apoia e incentiva comportamento de carreira adaptativos (e.g., exploração, planeamento), contribui para a adoção dos mesmos. Enquanto isso, a perceção de barreiras (e.g., falta de mestria) contribui de forma contrária, inibindo os mesmos (Lent et al., 1999; Lent \& Brown, 2013).

Para aceder a estes comportamentos de carreira, a literatura destaca múltiplas variáveis às quais se deve atender (e.g., exploração, autoeficácia, tomada de decisão) (e.g., Johnston, 2018; Rudolph et al., 2017), indo ao encontro das teorias mencionadas. Porém, a única medida específica para avaliar tais dimensões, já validada entre estudantes do secundário e universitário, é o Student Career Construction Inventory (SCCI, Savickas et al., 2018). Este inventário inclui um total de 18 itens distribuídos por quatro comportamentos adaptativos: cristalização do autoconceito vocacional, recolha de informação ocupacional, preparação para a tomada de decisão, e compromisso com uma escolha ocupacional. A SCCI, apesar de útil e fiel ao conceito de comportamentos de carreira adaptativos, não reflete uma visão ampla e integrativa dos contributos teóricos principais sobre tais constructos (e.g., as teorias TCC e TSCC). Uma medida alternativa recente que permite cumprir melhor este objetivo é a Escala de Desenvolvimento de Carreira de Universitários (EDCU), desenvolvida mais recentemente por Teixeira et al. (2019) para o contexto educativo brasileiro. Desenhada para fins de investigação e avaliação de intervenções de carreira, a EDCU procura atender à necessidade teórica de uma escala específica, baseada na teoria, que permita uma avaliação mais ampla e integradora de comportamentos de carreira adaptativos. Assim, a EDCU é composta por um total de 31 itens que pretendem medir comportamentos de exploração de carreira, tomada de decisão e identidade de carreira, identificados nas perspetivas desenvolvimentista e construtivista (Savickas, 2005; Super, 1980), e o locus de controlo e a autoeficácia, identificados na perspetiva sociocognitiva (Bandura, 1977; Lent \& Brown, 2013; Lent et al.,1999). A dimensão de exploração de carreira é definida como a procura por novas experiências, reflexão sobre si e sobre a própria carreira. A identidade é definida como a integração de aspetos profissionais à visão pessoal. A decisão de carreira é definida como a clareza na formulação dos objetivos profissionais. 0 locus de controlo é definido como a crença de que o sucesso se deve ao esforço da própria pessoa. Finalmente, a autoeficácia consiste na crença que as pessoas têm sobre as suas capacidades para lidar com uma determinada tarefa.

Dada a utilidade da EDCU para avaliar comportamentos de carreira adaptativos de forma rápida e integradora, o presente estudo pretende testar o modelo e estrutura dimensional proposto pelos autores da escala, bem como a sua precisão, numa amostra de estudantes universitários portugueses. Com base na literatura, espera-se obter um ajustamento do modelo de medida aos dados aceitável e valores de precisão satisfatórios com a EDCU, em universitários portugueses.

\section{MÉTODO}

\section{Participantes}

Os participantes foram recrutados com um método de amostragem não-probabilístico, por conveniência. De forma a responder aos objetivos do presente estudo, aleatorizou-se, posteriormente, os sujeitos por duas subamostras.

A subamostra A inclui um total de 111 estudantes universitários portugueses, com idades entre os 19 e os 35 anos $(M=22.67, D P=2.56)$, todos de etnia caucasiana $(100 \%)$ e $88(79.3 \%)$ mulheres. Analisando as áreas de estudo (Direção-Geral do Ensino Superior, 2018), 69 (62.2\%) encontravam-se a estudar na área de Direito, Ciências Sociais e Serviços; 11 (9.9\%) na área de Ciências; 11 (9.9\%) na área das Tecnologias; sete (6.3\%) na área da Saúde; seis (5.4\%) na área das Ciências da Educação e Formação de Professores; seis (5.4\%) na área Economia, Gestão e Contabilidade; e um (.9\%) na área de Arquitetura, Artes Plásticas e Design. A maioria dos participantes desta subamostra apresentou uma média de classificações escolares entre os 12 e os 15 valores $(n=77,69.4 \%)$ e uma minoria apresentou uma média superior a 18 valores $(n=1,0.9 \%)$.

A subamostra B é composta por um total de 105 estudantes universitários portugueses, com idades entre os 19 e os 36 anos $(M=22.42, D P=2.08)$, sendo a maioria de etnia caucasiana $(n=104 ; 99 \%)$ e mulheres ( $n=86 ; 81.9 \%$ ). Analisando as áreas de estudo (Direção-Geral do Ensino Superior, 2018), 64 (61\%) encontravam-se a estudar na área de Direito, Ciências Sociais e Serviços; 16 (15.2\%) na área das Tecnologias; oito (7.6\%) na área das Ciências; oito (7.6\%) na área de Economia, Gestão e Contabilidade; cinco (4.8\%) na área da Saúde; três ( $2.9 \%$ ) na área das Ciências da Educação e Formação de Professores; 
e um (1\%) na área de Arquitetura, Artes Plásticas e Design. A maioria dos participantes desta subamostra apresentou uma média de classificações escolares entre os 12 e os 15 valores $(n=73,69.5 \%)$ e uma minoria apresentou uma média superior a 18 valores $(n=2,1.9 \%)$.

\section{Medida}

A Escala de Desenvolvimento de Carreira de Universitários (EDCU) de Teixeira e colaboradores (2019) é uma escala composta por um total de 31 itens distribuídos por cinco subescalas consistentes. No estudo original, a subescala identidade de carreira é avaliada com seis itens (e.g., Item 2 "Consigo imaginar-me no futuro a trabalhar na profissão que escolhi"). 0 locus de controlo com cinco itens (e.g., Item 15 "Para eu me dar bem profissionalmente, vou precisar de contar com a ajuda da sorte"). A autoeficácia profissional é avaliada com nove itens (e.g., Item 20 "Domino as capacidades necessárias para exercer a minha profissão eficazmente"). A decisão de carreira com cinco itens (e.g., Item 10 "Tenho planos profissionais já bem estabelecidos"). E, a exploração ampliada com seis itens (e.g., Item 26 "Avalio constantemente quais as capacidades que preciso melhorar para atingir os meus objetivos profissionais"). A escala apresenta itens invertidos, nomeadamente os itens quatro ao seis, na subescala de identidade de carreira; os itens 12 ao 16, na subescala de locus de controlo; o item 11 da subescala de decisão de carreira; e, os itens 18, 21 e 24 da subescala de autoeficácia profissional (ver anexo 1). A escala de respostas é do tipo Likert e varia de 1 (a frase é totalmente falsa a seu respeito) a 5 (a frase é totalmente verdadeira a seu respeito) pontos.

No estudo original da escala, após o estudo piloto e a análise de re-teste com uma amostra independente, foi apreciado o ajustamento de dois modelos de medida aos dados. Os seus autores consideraram um modelo de medida penta-fatorial e um modelo hierárquico, no qual as respostas adaptativas foram consideradas como fator de segunda ordem. Os índices de ajustamento escolhidos pelos referidos autores foram os seguintes: Qui-quadrado com os respetivos graus de liberdade $\left(\chi^{2} / \mathrm{gl}\right)$; Comparative Fit Index (CFI); Root Mean Square Error Aproximation (RMSEA); Akaike Information Criterion (AIC); Bayes Information Criterion (BIC) e Browne-Cudeck Criterion (BCC). Ambos os modelos de medida apresentaram índices de ajustamento aceitáveis (modelo penta-fatorial: $\chi^{2} / \mathrm{gl}=2.73, \mathrm{GFI}=.82, \mathrm{CFI}=.82$, $\mathrm{RMSEA}=.07, \mathrm{AIC}=1305.00, \mathrm{BIC}=1318.29, \mathrm{BCC}=1559.81 ;$ modelo hierárquico: $\chi^{2} / \mathrm{gl}=2.49, \mathrm{GFI}=.82, \mathrm{CFI}$ $=.85, \mathrm{RMSEA}=.07, \mathrm{AIC}=1205.83, \mathrm{BIC}=1218.83, \mathrm{BCC}=1441.34)$. Contudo, o modelo hierárquico apresentou melhores índices de ajustamento.

\section{Procedimento}

Inicialmente, foi enviada uma mensagem por correio eletrónico aos autores da versão original da EDCU em português do Brasil, pedindo autorização para efetuar e analisar a versão para português de Portugal. Após receção da autorização, elaborou-se o projeto de investigação, o qual foi aprovado pelo comité de ética para a investigação em Ciências Sociais e Humanas da instituição proponente (parecer CEICSH 023/2019). De seguida, procedeu-se à revisão item a item da escala original. No final desta etapa, houve necessidade de analisar a escala no seio da equipa de investigação. Após haver acordo interjuízes, procedeu-se à reflexão falada da escala, com quatro dos participantes da amostra total, dois rapazes e duas raparigas. Desta forma, foi obtida a versão final, a qual foi enviada aos autores da escala original, para validação. Com intuito de tornar os itens compreensíveis, realizaram-se alguns ajustes simples (e.g., item 9 "Tenho metas definidas em relação à minha profissão e um plano para alcançá-las") e outros mais profundos (e.g., item 2 "Consigo imaginar-me no futuro a trabalhar na profissão que escolhi") relativamente à estrutura frásica, tendo em consideração as regras gramaticais da língua portuguesa (consultar Anexo1 e Teixeira et al., 2019).

A EDCU foi aplicada juntamente com um questionário sociodemográfico, no qual constavam perguntas sobre o sexo, a idade, a etnia, a área de estudo e a média de classificações no curso. Antes da administração do instrumento, contactaram-se diversas universidades de norte a sul de Portugal com o objetivo de obter autorização institucional para a realização do estudo. Posteriormente, investigadores de psicologia, com autorização dos diretores de curso e docentes das respetivas universidades, procederam à aplicação do instrumento em formato online através da plataforma Qualtrics, numa única sessão, de cinco a 10 minutos. De forma a controlar o fluxo de respostas, o instrumento deixou de estar disponível após o período de recolha pré-estabelecido.

\section{Análises}

Com a subamostra A, efetuaram-se análises fatoriais exploratórias (AFE) para determinar o número de fatores e respetivos itens com recurso ao software Statistical Package for the Social Science (IBM SPSS), versão 25.0 para Macintosh. Começou-se por verificar os pressupostos inerentes à análise, nomeadamente o valor do índice Kaiser-Meyer-Olkin (KMO) e do teste de esfericidade de Bartlett. Consideraram-se os valores de KMO superiores a .70 e de teste de Bartlett estatisticamente significativos, como adequados (Field, 2009). Os fatores foram extraídos através do método de Componentes Principais (principal 
component analysis - PCA) com rotação varimax, um método apropriado para o estudo de escalas cuja dimensionalidade e estrutura já foram objeto de estudos prévios (Field, 2009). A análise da solução fatorial teve como critérios de seleção principais: a matriz de correlação dos itens; a apreciação do teste de Cattell (1966); os eigenvalues de cada fator extraído, considerando valores iguais ou superiores a 1 , de acordo com o critério de Kaiser (Field, 2009); os valores de comunalidade, privilegiando-se valores superiores a 0.25 (Tabachnick \& Fidell, 2013); as saturações fatoriais com valores iguais ou superiores a .40; a contribuição da solução encontrada para a explicação da variância total do conjunto de itens ( $\geq 50 \%$ ); a coerência com o enquadramento teórico subjacente à medida e com a estrutura fatorial da EDCU definidas pelos autores da escala; e a simplicidade da solução fatorial. Em concordância com estes critérios de seleção, verificou-se ser necessário eliminar os itens 19, 20, 22 e 25, uma vez que apresentavam saturações fatoriais elevadas em mais do que três fatores em simultâneo, penalizavam a variância explicada pela solução fatorial e geravam incoerências com o enquadramento teórico e com a estrutura fatorial da EDCU no estudo original. Deste modo, considerou-se a solução retida com 27 dos 31 itens. Ainda nesta subamostra, foram efetuadas análises à consistência interna da escala, tendo em consideração a solução fatorial identificada. Para tal, consideraram-se os seguintes indicadores: Alfa de Cronbach $(\alpha)$, cujos valores superiores a .70 indicam boa precisão (Nunnally, 1970); Variância Média Extraída (VME), cujos valores iguais ou superiores a .50 indicam boa precisão (Fornell \& Larcker, 1981) e Confiabilidade Compósita (CC), cujos valores superiores ou iguais a .60 indicam boa precisão (Bagozzi \& Yi, 1988). A VME e a CC foram calculadas com recurso ao Microsoft Excel, versão 16.39 para Macintosh. As fórmulas aplicadas foram, respetivamente, $\sum\left(\lambda^{2}\right) /\left[\sum\left(\lambda^{2}\right)+\sum \varepsilon\right]$ e $\left(\sum \lambda\right)^{2} /\left[\left(\sum \lambda\right)^{2}+\sum \varepsilon\right]$ (Bagozzi \& Yi, 1988; Fornell \& Larcker, 1981).

Com a subamostra B, efetuaram-se, num segundo momento, análises fatoriais confirmatórias (AFC) com recurso ao software Analysis of Moment Structures (AMOS), versão 26.0 para Windows. Começou-se por verificar os pressupostos inerentes à análise: tamanho da amostra recomendado entre 100 a 200 participantes (Boomsma, 1985; Schumacker \& Lomax, 2010); normalidade multivariada (existente perante um coeficiente de Mardia $\leq 3$ ); ausência de outliers (existente perante a Distância de Mahalanobis com significância $>.001$ ); ausência de multicolinearidade (verificado perante $r<.90$ ); e presença de linearidade entre as variáveis (correlações estatisticamente significativas) (e.g., Garson, 2012; Marôco, 2010; Tabanick \& Fidell, 2013). Tendo-se verificado ausência de normalidade multivariada, recorreu-se à técnica de bootstrapping com 500 amostras (Gilson et al., 2013). Face à presença de um outlier, optou-se por efetuar a análise com e sem esta observação extrema (Pinto et al., 2013). No total, especificaram-se quatro modelos de medida. Dois modelos penta-fatoriais: o modelo 1 com 31 variáveis observadas e cinco variáveis latentes interrelacionadas (locus de controlo, autoeficácia profissional, exploração ampliada, identidade de carreira e decisão de carreira); e o modelo 3 com 27 variáveis observadas e as mesmas cinco variáveis latentes. Os restantes dois modelos consideraram uma estrutura hierárquica: o modelo 2 com 31 variáveis observadas, cinco fatores de primeira ordem (locus de controlo, autoeficácia profissional, exploração ampliada, identidade de carreira e decisão de carreira) e um fator de segunda ordem (respostas adaptativas); o modelo 4 com 27 variáveis observadas e os mesmos cinco fatores de primeira ordem e o mesmo fator segunda ordem. Para cada variável, fixou-se um peso fatorial em 1 e os erros foram estimados livremente (Oliveira et al., 2018). Posteriormente, efetuou-se a apreciação do ajustamento dos quatro modelos de medida aos dados com recurso aos seguintes índices: CFI e GFI, cujos valores superiores ou iguais a .90 indicam um bom ajustamento; RMSEA, considerado bom para valores entre .05 e .10; AIC, cujos valores menores indicam um melhor ajustamento; e o Bollen-Stine Chi-square Goodness of Fit Test com 500 amostras, considerado bom para valores de significância superiores a .05 (Gilson et al., 2013; Marôco, 2010; Oliveira et al., 2018).

\section{RESULTADOS}

No que concerne à AFE, os resultados indicaram adequabilidade da amostra (KMO $=.82)$ e que as correlações entre os itens eram suficientemente altas para se efetuar AFE, $\chi 2(351)=1688.51, p<.001$. Os resultados da PCA com rotação varimax permitiram identificar cinco eigenvalues cujos valores de Kaiser eram superiores a 1. Como apresentado na Tabela 1: o primeiro fator foi denominado exploração ampliada e inclui seis itens; o segundo fator foi denominado locus de controlo e inclui cinco itens; o terceiro fator foi denominado autoeficácia profissional e inclui cinco itens; o quarto fator foi denominado identidade de carreira e inclui seis itens; e o quinto fator foi denominado decisão de carreira e inclui cinco itens. A escala apresenta bons índices de precisão, apesar dos valores de VME para os três últimos fatores se encontrarem abaixo de .50 (Fornell \& Larcker, 1981). 
Tabela 1. Análise Fatorial Exploratória dos itens da EDCU: Pesos fatoriais (N=111)

\begin{tabular}{|c|c|c|c|c|c|}
\hline Itens & Fator 1 & Fator 2 & Fator 3 & Fator 4 & Fator 5 \\
\hline 1 & .19 & -.05 & -.15 & .68 & .21 \\
\hline 2 & .24 & -.01 & .16 & .71 & .11 \\
\hline 3 & .18 & -.09 & .07 & .66 & .32 \\
\hline 4 & .05 & .35 & .33 & .45 & -.14 \\
\hline 5 & -.11 & -.07 & .18 & .78 & .00 \\
\hline 6 & -.01 & .20 & .22 & .74 & .16 \\
\hline 7 & .26 & .06 & .09 & .23 & .63 \\
\hline 8 & .35 & .03 & .37 & .24 & .60 \\
\hline 9 & .41 & -.06 & .26 & .15 & .74 \\
\hline 10 & .34 & -.03 & .12 & .16 & .76 \\
\hline 11 & .07 & .34 & .46 & .02 & .46 \\
\hline 12 & -.08 & .83 & -.04 & -.04 & .19 \\
\hline 13 & .04 & .88 & .01 & .05 & -.03 \\
\hline 14 & -.02 & .78 & .09 & -.08 & .03 \\
\hline 15 & -.02 & .79 & .24 & .04 & -.04 \\
\hline 16 & .03 & .75 & .24 & .07 & -.07 \\
\hline 17 & .37 & -.24 & .59 & .14 & .28 \\
\hline 18 & -.03 & .14 & .70 & .13 & .26 \\
\hline 21 & .03 & .22 & .71 & .29 & .09 \\
\hline 23 & .41 & .05 & .62 & .15 & .25 \\
\hline 24 & -.12 & .22 & .77 & .00 & .00 \\
\hline 26 & .84 & .08 & -.03 & .05 & .15 \\
\hline 27 & .78 & .04 & .05 & .05 & .28 \\
\hline 28 & .66 & -.13 & -.03 & .07 & .13 \\
\hline 29 & .90 & .03 & .08 & .02 & .10 \\
\hline 30 & .85 & .03 & .06 & .10 & .17 \\
\hline 31 & .44 & -.08 & .15 & .23 & .24 \\
\hline Alfa de Cronbach $(\alpha)$ & .87 & .88 & .80 & .78 & .84 \\
\hline VME & .58 & .65 & .46 & .46 & .42 \\
\hline $\mathrm{CC}$ & .89 & .90 & .81 & .83 & .78 \\
\hline Eigenvalue & 7.50 & 4.24 & 2.61 & 1.78 & 1.11 \\
\hline \% de variância & 16.41 & 30.39 & 42.02 & 53.59 & 63.8 \\
\hline
\end{tabular}

Nota. Os valores apresentados a negrito representam o fator principal onde o item satura. VME = Variância Média

Extraída; $\mathrm{CC}=$ Confiabilidade Compósita

Prosseguindo o estudo, efetuaram-se análises de correlação de Pearson entre as subescalas, considerando os 27 itens, valores mínimos, máximos, médias e desvios-padrão (tabela 2). As subescalas apresentam correlações positivas e estatisticamente significativas entre si, exceto a subescala do locus de controlo. Assim, o pressuposto da linearidade não está cumprido, apenas para algumas relações do locus de controlo com outras subescalas (e.g., exploração ampliada). Contudo, optou-se por manter esta subescala na análise, uma vez que se verificam relações entre o locus de controlo e a autoeficácia, bem como com o fator de segunda ordem (Levers-Landis et al., 2011). Os resultados apontam para correlações positivas e moderadas $(.30<r<.50$, Cohen, 1998) entre a identidade - decisão de carreira, decisão de carreira - exploração ampliada, autoeficácia profissional - decisão de carreira e autoeficácia profissional identidade de carreira. Assim, a adoção de mais comportamentos de tomada de decisão pode ser incentivada por uma identidade de carreira fortalecida pela adoção de atitudes de carreira e por crenças de autoeficácia profissional. Além disso, esta última também parece contribuir para uma melhor conciliação entre os valores e interesses do próprio e o percurso de carreira escolhido. 
Tabela 2. Correlações de Pearson, mínimos, máximos, média e desvio-padrão na subamostra B (N=105).

\begin{tabular}{|c|c|c|c|c|c|c|c|c|c|c|}
\hline & EDCU & IC & DC & LC & EA & AP & Min. & Máx. & $M$ & $D P$ \\
\hline EDCU & . & & & & & & 2.19 & 4.74 & 3.64 & 0.48 \\
\hline IC & $.69^{* * *}$ & . & & & & & 1.83 & 5.00 & 3.99 & 0.74 \\
\hline DC & $.73^{* * *}$ & $.47^{* * *}$ & . & & & & 1.20 & 5.00 & 3.50 & 0.82 \\
\hline LC & $.41^{* * *}$ & .08 & -.02 & . & & & 1.40 & 5.00 & 3.57 & 0.81 \\
\hline EA & $.60^{* * *}$ & .10 & $.46^{* * *}$ & .04 & . & & 1.67 & 5.00 & 3.63 & 0.73 \\
\hline $\mathrm{AP}$ & $.73^{* * *}$ & $.50 * * *$ & $.40^{* * *}$ & $.23^{*}$ & $.26^{* *}$ & . & 1.80 & 5.00 & 3.42 & 0.74 \\
\hline
\end{tabular}

Nota. EDCU = Escala de Desenvolvimento de Carreira; IC = Identidade de Carreira; DC = Decisão de Carreira; LC = Locus de Controlo; EA = Exploração Ampliada; AP = Autoeficácia Profissional

${ }^{*} p<.05 ;{ }^{* *} p<.01 ;{ }^{* * *} p<.001$

Posteriormente, através de AFC, testaram-se os quatro modelos de medida. Os valores dos índices de ajustamento dos modelos de medida encontram-se especificados na Tabela 3.

Tabela 3. Índices de ajustamento para os modelos de medida especificados: N = 105 .

\begin{tabular}{|c|c|c|c|c|c|}
\hline & AIC & GFI & CFI & RMSEA & Bollen-Stine $p$ value \\
\hline \multicolumn{6}{|c|}{ Modelos com outliers $(N=105)$} \\
\hline Modelo 1 & 843.93 & .71 & .82 & .08 & n.s \\
\hline Modelo 2 & 854.24 & .70 & .81 & .08 & n.s. \\
\hline Modelo 3 & 655.78 & .74 & .83 & .08 & n.s. \\
\hline Modelo 4 & 669.39 & .73 & .82 & .08 & $*$ \\
\hline \multicolumn{6}{|c|}{ Modelos sem outliers $(N=104)$} \\
\hline Modelo 1 & 846.89 & .70 & .82 & .08 & n.s. \\
\hline Modelo 2 & 858.23 & .69 & .81 & .08 & $*$ \\
\hline Modelo 3 & 658.46 & .73 & .83 & .08 & $*$ \\
\hline Modelo 4 & 673.43 & .72 & .82 & .09 & $*$ \\
\hline
\end{tabular}

Nota. Modelo1 = Modelo com 31 variáveis observadas e cinco variáveis latentes interrelacionadas; Modelo $2=$

Modelo com 31 variáveis observadas, cinco fatores de primeira ordem e um fator de segunda ordem; Modelo $3=$ Modelo com 27 variáveis observadas e cinco variáveis latentes interrelacionadas; Modelo $4=$ Modelo com 27 variáveis observadas, cinco fatores de primeira ordem e um fator de segunda ordem.

$* p<.05$

Os valores de RMSEA indicam um bom ajuste dos modelos de medida aos dados. Porém, o índice GFI aponta para um fraco ajustamento (valores inferiores a .80) e os valores de CFI apontam para um ajustamento sofrível (valores entre .80 e .90) (Marôco, 2010). Para além disso, a comparação dos resultados derivados das análises com e sem o único outlier detetado indica a existência de eventual enviesamento dos resultados na presença desse outlier, sendo, por isso, de privilegiar os resultados sem outlier. Os modelos de medida sem o outlier apresentaram índices de ajustamento moderadamente desejáveis em termos estatísticos. Não se apresentam resultados decorrentes dos índices de modificação sugeridos pelo AMOS, uma vez que estes remetiam para correlação entre erros - opção estatística pouco recomendada (Marôco, 2010; Tabachnick \& Fidell, 2013) - e, mesmo testando os modelos com correlações entre erros, os índices de ajustamento mantiveram-se globalmente idênticos.

Assim, entre os resultados sem outlier, quando se comparam os índices de ajustamento dos quatro modelos de medida, verifica-se que o ajustamento é melhor para o modelo 3 com 27 variáveis observadas e cinco variáveis latentes interrelacionadas (AIC $=658.46, \mathrm{GFI}=.73, \mathrm{CFI}=.83, \mathrm{RMSEA}=.08$ ) (Figura 1). 


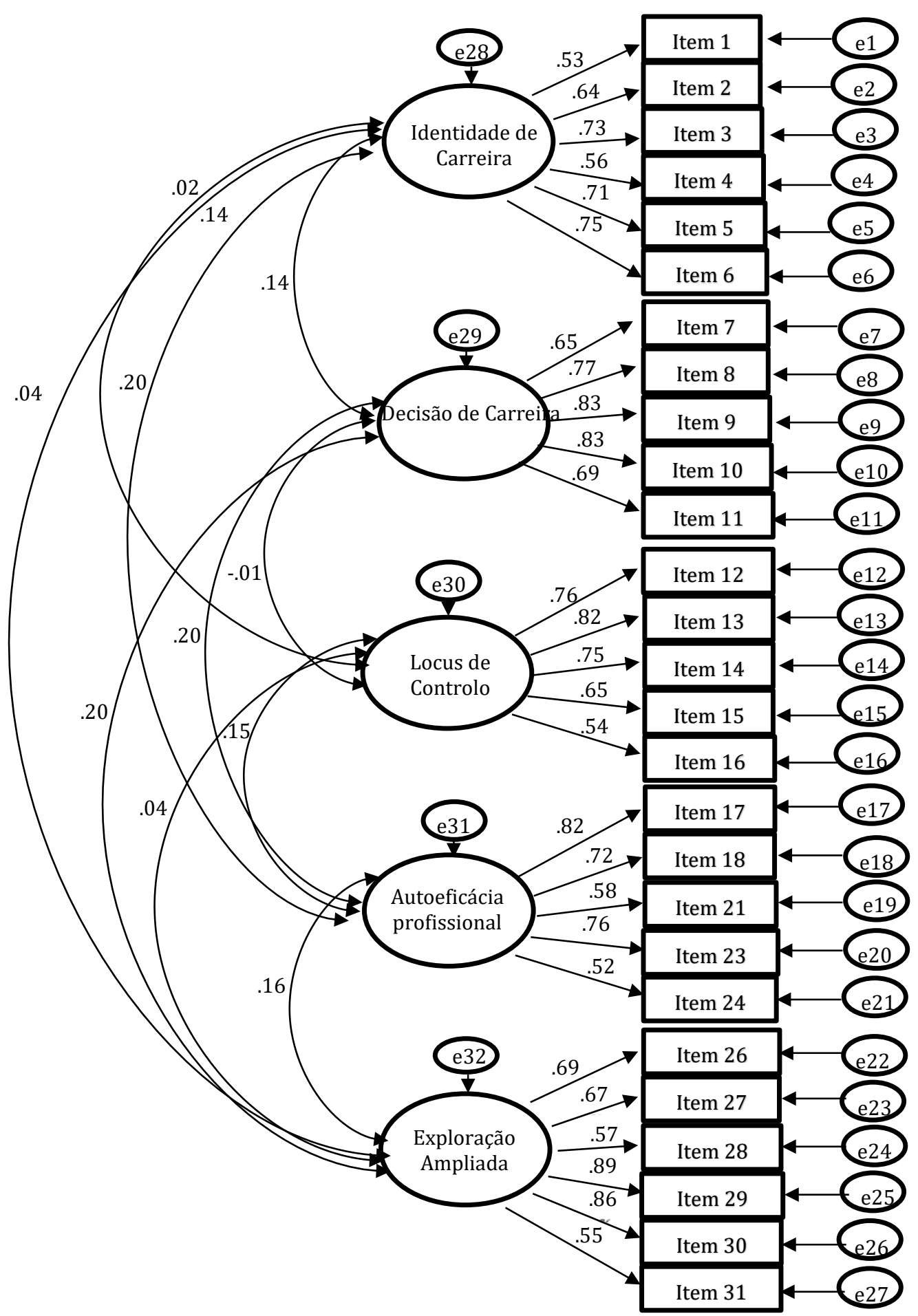

Figure 1. Pesos fatoriais padronizados do modelo de medida 3, com 27 variáveis observadas (à direita) e cinco variáveis latentes interrelacionadas (à esquerda). Pesos resultantes na AFC efetuada na subamostra B sem outliers $(\mathrm{N}=104)$.

\section{DISCUSSÃo}

O objetivo deste estudo consistiu em testar a estrutura dimensional e a precisão da EDCU numa amostra de estudantes universitários portugueses, de forma a que investigações e intervenções de carreira futuras pudessem fazer uso da escala em Portugal. Com base na literatura, equacionou-se que a escala apresentaria índices de ajustamento aos dados aceitáveis e boa precisão. Os resultados indicam índices de ajustamento da medida aos dados moderadamente satisfatórios e bons índices de precisão, apoiando essa hipótese de investigação. 
Os índices de ajustamento obtidos na AFC para os modelos 1 e 2 na amostra portuguesa, com 31 variáveis observadas, apresentaram-se ligeiramente abaixo dos índices encontrados no estudo original (Teixeira et al., 2019). Ao complementar esses resultados com os derivados da AFE, tal parece ser compreensível pela sobreposição fatorial de alguns itens. Assim, após se eliminarem quatro itens, obtiveram-se tanto uma solução fatorial mais simples na AFE, como índices de ajustamento mais desejáveis. Ainda que neste estudo se tenha verificado melhor ajustamento do modelo dos cinco fatores interrelacionados (modelo 3) aos dados da presente amostra, reconhece-se a pertinência de replicar este trabalho a partir de outras amostras de maior dimensão. Assim, será possível obter evidência adicional para clarificar se o modelo penta-fatorial prevalece o melhor ajustado aos dados de outros estudantes universitários, ou se haverá possibilidade de considerar o modelo de medida hierárquico. Neste estudo, os valores dos índices foram semelhantes entre o modelo de medida penta-fatorial e o modelo hierárquico, embora melhores para o primeiro. Contudo, estudos próximos poderão ajudar a perceber a viabilidade de considerar um modelo de medida hierárquico. A utilização de um modelo hierárquico apresenta vantagens tanto para a investigação, como para a prática. Estes são mais flexíveis na sua utilização e parcimoniosos na explicação das covariâncias entre os fatores de primeira ordem (Oliveira et al., 2018). Assim, poderão tornar a interpretação das pontuações derivadas de uma medida mais simples e facilitar a avaliação das respostas adaptativas no modelo de adaptação à carreira (Savickas, 2005; Savickas \& Porfeli, 2012).

Embora os resultados deste estudo contribuam para a teoria e para a prática psicológica de carreira, é importante considerar as suas limitações. Primeiro, é de notar que a AFC é sensível ao tamanho da amostra e à complexidade do modelo de medida (Boomsma, 1985; Schumacker \& Lomax, 2010), o que significa, por exemplo, que os valores dos índices de ajustamento podem ser mais baixos em amostras de reduzida dimensão. Como tal, é importante que futuros estudos repliquem as análises, recorrendo a uma amostra mais alargada de estudantes universitários portugueses. Em segundo lugar, é importante notar que, ao contrário do SCCI (Savickas et al., 2018), a EDCU inclui a dimensão de autoeficácia (Teixeira et al., 2019). A explicação partilhada pelos autores do SCCI para a exclusão deste constructo, é que a autoeficácia é uma dimensão situada entre os recursos adaptativos e as respostas adaptativas. Com efeito, não só seria pertinente validar o SCCI junto de estudantes universitários portugueses, como também seria importante comparar as medidas de forma a aprofundar o papel da autoeficácia nas respostas adaptativas e verificar qual a medida que melhor se ajusta à população portuguesa. Não obstante, ao reconhecer a importância da convergência entre teorias da carreira (Savickas, 2002), a EDCU parece constituir um instrumento promissor para apoiar práticas de avaliação psicológica de carreira coerentes com uma perspetiva teórica integradora. Tal poderá ser igualmente tido em conta em estudos comparativos das propriedades psicométricas do SCCI e da EDCU em Portugal. Poderá também ser considerado ao nível da eventual disponibilização futura dessas medidas a psicólogos, para que possam selecionar aquela que, além de apresentar robustez psicométrica, esteja também mais alinhada com a sua opção teórica.

Quanto a investigações futuras, estas poderiam ainda analisar a validade da EDCU por relação a outras variáveis (e.g., o autoconceito, os interesses profissionais e as expectativas de resultados no âmbito da carreira), bem como testar a sua estabilidade temporal através de um estudo de cariz longitudinal. A partir de estudos psicométricos complementares da EDCU, seria possível consolidar as suas propriedades e prosseguir, por exemplo, em estudos luso-brasileiros de comportamentos adaptativos de carreira e em estudos comparativos de grupos (e.g., estudantes do ensino superior no norte, centro, sul e arquipélagos de Portugal).

Em síntese, os resultados do presente estudo apontam para a utilidade da EDCU em traduzir respostas adaptativas de estudantes universitários portugueses, algo igualmente verificado com estudantes brasileiros (Teixeira et al., 2019). Além disso, embora sejam necessários mais estudos psicométricos em Portugal e noutros países, os resultados do presente estudo permitem dar continuidade à literatura sobre o modelo de adaptação à carreira, tanto em Portugal, como no Brasil. Inclusive, a escala poderá ser utilizada na prática psicológica, como meio de avaliação do impacto de práticas de aconselhamento de carreira de universitários.

\section{REFERÊNCIAS}

Ambiel, R. A. (2014). Adaptabilidade de Carreira: Uma Abordagem Histórica de Conceitos, Modelos e Teorias. Revista Brasileira de Orientação Profissional, 15(1), 15-24.

Bagozzi, R. P., \& Yi, Y. (1988). On the evaluation of structural equation models. Journal of the academy of marketing science, 16(1), 74-94.

Bandura, A. (1977). Self-efficacy: Toward a Unifying Theory of Behavioral Change. Psychological Review, 84(2), 191-215. https://doi.org/10.1037/0033-295X.84.2.191

Bandura, A. (1986). Social foundations of thought and action. Englewood Cliffs. 
Bardagi, M., \& Albanaes, P. (2015). Relações entre adaptabilidade de carreira e personalidade: Um estudo com universitários ingressantes brasileiros. Psicologia, 29, 35-44. https://doi.org/10.17575/rpsicol.v29i1.989

Boomsma, A. (1985). Nonconvergence, Improper Solutions, and Starting Values in LISREL Maximum Likelihood Estimation. Psychometrika, 50, 229-242. https://doi.org/10.1007/bf02294248

Cattell, R.B. (1966). The scree test for the number of factors. Multivariate Behavioral Research,1, 245-276. https://doi.org/10.1207/s15327906mbr0102_10

Cohen, J. E. (1988). The significance of a product moment rs. In J. Cohen (Ed), Statistical Power Analysis for the Behavioral Sciences (pp. 75-105). Lawrence Erlbaum Associates.

Direção-Geral do Ensino Superior. (2018). Índice por área de estudo e curso. http://www.dges.gov.pt/guias/indarea.asp?area=II

Duarte, D. M., Soares, C. M., Fraga, S., Rafael, M., Lima, R. M., \& Paredes, I. ... \& Djaló, A. (2012). Career AdaptAbilities Scale-Portugal Form: Psychometric properties and relationships to employment status. Journal of Vocational Behavior, 80, 725-729. https://doi.org/10.1016/j.jvb.2012.01.019

Field, A. (2009). Discovering statistics using IBM SPSS statistics (3rd ed.). SAGE.

Fornell, C., \& Larcker, D. F. (1981). Evaluating structural equations models with unobservable variables and measurement error. Journal of Marketing, 18(1), https://doi.org/10.1177/002224378101800104

Garson, D. (2012). Testing Statistical Assumptions. Statistical Associates Publishing

Gilson, K.M., Bryant, C., Bei, B., Komiti, A., Jackson, H., \& Judd, F. (2013). Validation of the Drinking Motives Questionnaire (DMQ) in older adults. Addictive Behaviors, 38, 2196-2202. https://doi.org/10.1016/j.addbeh.2013.01.021

Gomes, C. (2017). O processo de adaptação na carreira: Estudo com finalistas universitários portugueses [Dissertação de Mestrado, Universidade do https://repositorium.sdum.uminho.pt/bitstream/1822/49379/1/Cláudia\%20Correia\%20Ramos \%20Gomes.pdf

Hirschi, A., Herrmann, A., \& Keller, A. C. (2015). Career Adaptivity, Adaptability, and Adapting: A conceptual and Empirical Investigation.Journal of Vocational Behavior, 87, 1-10. https://doi.org/10.1016/j.jvb.2014.11.008

Johnston, C. S. (2018). A systematic review of the career adaptability literature and future outlook. Journal of Career Assessment, 26(1), 3-30. https://doi.org/10.1177/1069072716679921

Lent, R., \& Brown, S. (2013). Social cognitive model of career self-management: Toward a unifying view of adaptive career behavior across the life span. Journal Of Counseling Psychology, 60(4), 557-568. https://doi.org/10.1037/a0033446

Lent, R., Hackett, G., \& Brown, S. (1999). A Social Cognitive View of School-to-Work Transition. The Career Development Quarterly, 47(4), 297-311. https://doi.org/10.1002/j.2161-0045.1999.tb00739.x

Levers-Landis, C. E., Burant, C. J., \& Hazen, R. (2011). The Concept of Bootstrapping of Structural Equation Models with Smaller Samples: An Illustration Using Mealtime Rituals in Diabetes Management. Journal of Developmental \& Behavioral Pediatrics, 32(8), 619-626.

Marôco, J. (2010). Análise de Equações Estruturais: Fundamentos teóricos, Software \& Aplicações. Edições Report Number.

Monteiro, S., \& Almeida, L.S. (2015). The relation of career adaptability to work experience, extracurricular activities, and work transition in Portuguese graduate students. Journal of Vocational Behavior, 91, 106-112. https://doi.org/10.1016/j.jvb.2015.09.006

Nunnally, J. C. (1970). Introduction to psychological measurement. McGraw-Hill.

Oliveira, E. P., Merino, M. D., Privado, J., \& Almeida, L. S. (2017). Funcionamento psicológico positivo numa amostra portuguesa de estudantes. Revista de Estudios e Investigación en Psicología y Educación, 7, 93-96. https://doi.org/10.17979/reipe.2017.0.07.2733

Oliveira, I. M, Taveira, M. C, Porfeli, E. J., \& Grace, R. C. (2018). Confirmatory study of the Multidimensional Scales of Perceived Self-Efficacy with children. Universitas Psychologica, 17, 1-12. https://doi.org/10.11144/javeriana.upsy17-4.csms

Oliveira, M. C. (2014). Sucesso na Carreira Depois da Graduação: Estudo Longitudinal Prospetivo da Transição Universidade-Trabalho [Tese de Doutoramento, Universidade de São Paulo]. http://www.teses.usp.br/teses/disponiveis/59/59137/tde-13112014-101423/pt-br.php

Oliveira, M. C. D., \& Dela Coleta, M. F. (2008). Adaptação e validação da Escala Combinada de Atitudes da Maturidade de Carreira (CDA): versão para estudantes universitários. Revista Brasileira de Orientação Profissional, 9(2), 45-65.

Perrone, L. \& Vickers, M. H. (2003). Life after graduation as a "very uncomfortable world": An Australian case study. Education + Training, 45(2), 69-78. 
Pinheiro, M. (2017). Transição Universidade-Emprego: Relações entre Prontidão Adaptativa, Respostas Adaptativas e Empregabilidade percebida [Dissertação de Mestrado, Universidade do Minho]. https://repositorium.sdum.uminho.pt/bitstream/1822/46531/1/Marco\%20Pinheiro\%20A68812 .pdf

Pinto, J. C., Taveira, M. C., Candeias, A., \& Araújo, A. (2013). Análise fatorial confirmatória da Prova de Avaliação de Competência Social Face à Carreira. Psicologia: Reflexão e Crítica, 26 (3), 469-478.

Postigo, F. L. J. \& Oliveira, M. C. (2015). A experiência da transição universidade-trabalho: relatos de recémformados brasileiros. Revista Amazônica, 16 (2), 289-310.

Rudolph, C.W., Lavigne, K.N., \& Zacher, H. (2017). Career adaptability: A meta-analysis of relationships with measures of adaptivity, adapting responses, and adaptation results. Journal of Vocational Behavior, 98, 17-34. https://doi.org/10.1016/j.jvb.2016.09.002

Savickas, M. L. (2002). Career construction: A developmental theory of vocational behavior. In D. Brown (Ed.), Career choice and development (pp. 149-205). Jossey Bass.

Savickas, M. L. (2005). The theory and practice of career construction. In Brown, S. D. \& Lent, R.W (Eds.). Career development and counseling: Putting theory and research to work, (pp. 42-70). John Wiley \& Sons Inc.

Savickas, M. L. (2013). The theory and practice of career construction. In S. D. Brown \& R. W. Lent (Eds.), Career development and counselling: Putting theory and research to work (2nd ed., pp. 147-183). Wiley.

Savickas, M.L. \& Porfeli, E.J. (2012). Career Adapt-Abilities Scale: Construction, reliability, and measurement equivalence across 13 countries. Journal of Vocational Behavior, 80, 661-673. https://doi.org/10.1016/j.jvb.2012.01.011

Savickas, M. L., Porfeli, E. J., Hilton, T. L., \& Savickas, S. (2018). The Student Career Construction Inventory. Journal of Vocational Behavior, 106, 138-152. https://doi.org/10.1016/j.jvb.2018.01.009

Schoon, I. \& Silbereisen, R. K. (20009). Conceptualizing school-to-work transition in context. In I. Schoon \& R. K. Silbereisen (Eds.), Transition from school-to-work: Globalization, individualization and patterns of diversity (pp. 3-30). Cambridge University Press.

Schumacker, R. E. \& Lomax, R. G. (2004). Correlation. In Lomax, R. G. \& Schumacker, R. E. (Eds.) A Beginner's Guide to Structural Equation Modeling (pp.33- 51). Routledge Taylor \& Francis Group.

Sidiropoulou-Dimakakou, D., Mylonas, K., Argyropoulou, K., \& Tampouri, S. (2012). Career Decision-making Difficulties, Dysfunctional Thinking and Generalized Self-Efficacy of University Students in Greece. World Journal of Education, 2(1), 117-130.

Super, D. E. (1980). A life-span, life-space approach to career development. Journal of Vocational Behavior, 16(3), 282-298.

Tabachnick, B. \& Fidell, L. (2013). Using Multivariate Statistics. Pearson Education, Inc.

Teixeira, M. A. P., \& Gomes, W. B. (2004). Estou me formando...E agora? Reflexões e Perspetivas de jovens formandos universitários. Revista Brasileira de Orientação Profissional, 5(1), 47-62.

Teixeira, M. A. P., Oliveira, M. P., Melo-Silva, L. L., \& Taveira, M. C. (2019). Escala de Desenvolvimento de Carreira de Universitários: construção, características psicométricas e modelo das respostas adaptativas. Revista Psicologia: Organizações e Trabalho, 19, 703-712.

Wilhelm, F., \& Hirschi, A. (2019). Career self-management as a key factor for career wellbeing. In I. L. Potgieter, N. Ferreira \& M. Coetzee (Eds.). Theory, Research and Dynamics of Career Wellbeing (pp. 132). Switzerland: Springer. https://doi.org/10.31234/osf.io/9w36u

World Economic Forum (2018). The future of Jobs Report 2018. http://www3.weforum.org/docs/WEF_Future_of_Jobs_2018.pdf

$\begin{array}{ll}\text { Historial do artigo } & \\ \text { Recebido } & 08 / 2019 \\ \text { Aceite } & 01 / 2021 \\ \text { Publicado online } & 03 / 2021 \\ \text { Publicado } & 06 / 2021\end{array}$




\section{ANEXOS}

Anexo 1. Escala de Desenvolvimento de Carreira de Universitários - versão portuguesa integral

Por favor, responda aos itens abaixo marcando o número que melhor representa a sua opinião. A escala de resposta varia de 1 (a frase é totalmente falsa a seu respeito) a 5 (a frase é totalmente verdadeira a seu respeito). Preencha o espaço que melhor representa o modo como pensa, sente e age.

\begin{tabular}{|c|c|c|c|c|c|}
\hline Itens & 1 & 2 & 3 & 4 & 5 \\
\hline 1.Trabalhar na profissão que escolhi é muito importante para a minha realização pessoal. & & & & & \\
\hline 2.Consigo imaginar-me no futuro a trabalhar na profissão que escolhi. & & & & & \\
\hline 3.Sinto-me satisfeito(a) e tranquilo(a) com a minha opção profissional. & & & & & \\
\hline 4.Não me sinto motivado(a) com a carreira profissional que escolhi. & & & & & \\
\hline $\begin{array}{l}\text { 5.Dou comigo a imaginar se outras profissões não estariam mais de acordo com os meus } \\
\text { interesses e valores. }\end{array}$ & & & & & \\
\hline 6.Tenho dúvidas se realmente quero fazer carreira na profissão que escolhi. & & & & & \\
\hline 7.Considero que tenho um projeto profissional realista. & & & & & \\
\hline 8.É claro para mim quais são os meus objetivos profissionais. & & & & & \\
\hline 9.Tenho metas definidas em relação à minha profissão e um plano para alcançá-las. & & & & & \\
\hline 10.Tenho planos profissionais já bem estabelecidos. & & & & & \\
\hline 11.Tenho dificuldades em definir um plano profissional para mim. & & & & & \\
\hline $\begin{array}{l}\text { 12. Conseguir uma boa posição na minha profissão depende de acontecimentos que estão } \\
\text { fora do meu controlo. }\end{array}$ & & & & & \\
\hline 13.Considero que o meu futuro profissional depende de forças externas ao meu controlo. & & & & & \\
\hline $\begin{array}{l}\text { 14.As minhas oportunidades profissionais dependem mais do mercado de trabalho ou dos } \\
\text { outros do que de mim próprio(a). }\end{array}$ & & & & & \\
\hline 15.Para me dar bem profissionalmente, vou precisar de contar com a ajuda da sorte. & & & & & \\
\hline $\begin{array}{l}\text { 16.Não há muita coisa que posso fazer para aumentar as minhas chances de sucesso } \\
\text { profissional. }\end{array}$ & & & & & \\
\hline $\begin{array}{l}\text { 17.Considero-me um(a) profissional bem preparado(a) na área em que atuo ou pretendo } \\
\text { atuar. }\end{array}$ & & & & & \\
\hline $\begin{array}{l}\text { 18.Acho que não tenho conhecimentos suficientes para exercer a minha profissão } \\
\text { satisfatoriamente. }\end{array}$ & & & & & \\
\hline 21.Sinto-me inseguro(a) para exercer a minha profissão. & & & & & \\
\hline $\begin{array}{l}\text { 23.Sinto-me capaz de executar satisfatoriamente as tarefas relacionadas com a minha } \\
\text { profissão. }\end{array}$ & & & & & \\
\hline 24.Sinto que tenho ou terei dificuldades para desempenhar bem o meu papel profissional. & & & & & \\
\hline $\begin{array}{l}\text { 26. Avalio constantemente quais as capacidades que preciso melhorar para atingir os meus } \\
\text { objetivos profissionais. }\end{array}$ & & & & & \\
\hline $\begin{array}{l}\text { 27.Tenho refletido sobre as minhas experiências para identificar ou refinar os meus } \\
\text { interesses relacionados com a profissão. }\end{array}$ & & & & & \\
\hline 28.Procuro ativamente informações sobre oportunidades de trabalho na minha profissão. & & & & & \\
\hline $\begin{array}{l}\text { 29.Tento identificar possíveis barreiras ou dificuldades para executar os meus planos } \\
\text { profissionais. }\end{array}$ & & & & & \\
\hline 30.Tenho explorado formas de lidar com possíveis barreiras aos meus planos profissionais. & & & & & \\
\hline $\begin{array}{l}\text { 31.Tento construir e manter uma rede de contatos pessoais que me possam ajudar a } \\
\text { ingressar no mercado de trabalho. }\end{array}$ & & & & & \\
\hline
\end{tabular}

